\title{
When to Advise Medical Treatment for Open-Angle Glaucoma
}

\section{STEPHEN M. DRANCE}

Vancouver, Canada

The decision to treat open-angle glaucoma when the disease has manifested signs of damage is universally accepted. Unfortunately the treatment of the disease usually only addresses pressure reduction in the hope and expectation that this alone will slow down or arrest the disease. In many places the initial attempt to reduce pressure is medical and I will therefore not address the question as to whether medical or surgical treatment should come first.

I will deal with difficult problem as to when to start therapy in the open angle glaucoma suspect, who by definition has no definite evidence of glaucomatous damage.

Various population surveys showed that there is a number of people with slight elevations of intraocular pressure who might be subjected to unnecessary inconvenience and toxic side-effects. The term 'ocular hypertension' was introduced epidemiologically to describe those patients who have elevations of intraocular pressure without any evidence of tissue disturbance of the optic nerve head or visual field defects. The term, unfortunately, caught on clinically and has now even been used as 'benign ocular hypertension' by some, which is certainly a misnomer as eyes with elevations of intraocular pressure carry an increased risk of subsequently developing damage and therefore should not be considered benign. An attitude has also developed that 'ocular hypertension' is a separate disease distinct from chronic open-angle glaucoma. This concept cannot be defended by any of the known facts and the introduction of this epidemiologically useful label has caused a therapeutic nihilism among ophthal- mologists which is not warranted. The term 'ocular hypertension' in fact implies, in the definition, no tissue damage at the optic nerve head and no visual field defect and it is therefore essential that the examination of the optic nerve must be meticulously carried out and the visual field reliably examined to make sure that both of these are indeed completely normal before the term is even used. Computerised perimetry, when properly used and interpreted, has provided more reliable assessment of the visual field but the newer, more sensitive techniques of optic disc analysis and retinal nerve fibre layer evaluations have not yet found universal acceptance. It is therefore almost certain that early damage is often missed.

In order to present this subject briefly, I will make some assumptions, all of which bear critical examination and might form the basis of a separate symposium.

The assumptions are as follows:

(1) Reduction of elevated intraocular pressure prevents damage from taking place in an undamaged eye;

(2) Reduction of intraocular pressure halts or slows the progression of damage to the optic nerve;

(3) Means are not yet available to identify reliably, those early field defects and disc abnormalities which are reversible;

(4) The agents which we use in the treatment of glaucoma are not in themselves harmful to the eye.

Even assuming the above to be true, the issue which we face is whether all patients with elevated intraocular pressures require pres- 
sure reduction or whether one should identify those at risk of developing damage when the risk benefit ratio would be optimal. The past two decades have clearly shown that not all those whose pressures are elevated are at risk of developing the disease. We know that elevated intraocular pressure is undoubtedly a damaging factor from patients with angle closure glaucoma and the secondary glaucomas who develop damage identical to chronic simple glaucoma. In them, elevated intraocular pressure is probably the only operative factor. Experiments in primates also suggest the same damaging sequences when intraocular pressure is artificially elevated. Elevated intraocular pressure therefore is undoubtedly a risk factor and may in fact be the major risk factor. The higher the intraocular pressure therefore the greater the risk of optic nerve damage. There is some evidence that it is not the maximum pressure but the fluctuations in intraocular pressure which are more likely to be damaging. A rising intraocular pressure may also be important as the optic nerve head may be able to withstand a given level of intraocular pressure but a rising intraocular pressure does not provide the assurance that the susceptibility threshold of the nerve will not be passed by the rising pressure. It would help if we could test the susceptibility accurately. With a high intraocular pressure there is an additional risk that corneal decompensation or retinal vascular occlusions may develop.

It has also become clear that low tension glaucoma is not as uncommon as we once believed. Total population surveys identify many such patients. Even the exclusion of those in whom there are diurnal pressure rises and those in whom the intraocular pressures, although still statistically normal, are in the upper normal range and those who may have intermittent intraocular pressure elevations, there is still a sizeable group of individuals who develop classical progressive glaucoma damage and in whom pressures in the midteens are the highest ever recorded. These individuals, on ocular examination, are clinically indistinguishable from the classical glaucoma so that glaucomatous damage can obviously occur in the absence of an elevated intraocular pressure. We should ask ourselves why such individuals are so susceptible to normal intraocular pressure and whether intraocular pressure in them plays any part at all.

These other risk factors are of two kinds. Firstly, those which show the earliest signs of tissue and function damage and which, in fact, would not be risk factors at all but signs of early disturbance. Among them are the disturbances of colour vision, the changes of contrast sensitivity, both spatial and temporal, the interference with the light sense manifested as an increased scatter of responses or an overall diminution of the light sense itself. These problems are being addressed by some of the new automatic perimeters. The patterned electro-retinograms and even the visual evoked potentials may show similar disturbance. On the structural side, a diminution of the neuroretinal rim area, the presence of haemorrhages on the disc and retinal nerve fibre losses all indicate that early damage exists.

Secondly, there are risk factors which predict that an individual is likely to develop damage which is not yet demonstrable. The predictive factors include age and diabetes, the presence of coronary disease and vascular hypertension, the presence of an impending or an intermittent hypotensive episode, the presence of vasospastic disease such as migraine and many of its variants, and also rheological abnormalities of the blood. There are also genetic factors, such as a family history of glaucoma or diabetes and even of stroke.

Finally, we have learned to suspect myopia as a risk factor and the fellow eye of an individual who has the disease in one eye has a much greater chance of developing damage.

A study of all risk factors (and we are probably only identifying some of them in this multifactorial disease) should ultimately allow us to assess an individual's chance of developing the disease in fairly accurate terms, such as can be done with stepwise discriminant analysis. In the future we may in fact be able to identify more precisely the individuals who should be treated and in whom the benefits of the therapy outweigh the risks and inconvenience and the cost of such therapy. 